\title{
Bericht der Kommission für das Wörterbuch der deutschen Rechtssprache, fiir das Jahr 1906.
}

\author{
Von Heinrich Brunner.
}

Abgedruckt aus dem Jahresbericht der Hermann und Elise geb. Heckmann Wentzel-Stiftung für 1906.

Eine Zusammenkunft der Kommissionsmitglieder ist in verflossenen Jahre nicht veranstaltet worden. Die nächste Tagung der Kommission soll Ostern 1907 in Heidelberg stattfinden. Die Arbeiten für das Rechtswörterbuch sind nach Maßgabe der verfügbaren Mittel fortgesetzt worden. Über das Ergebnis hat der wissenschaftliche Leiter des Unternehmens den hier folgenden Jahresbericht erstattet.

Bericht des Hrn. Schröder.

Die Sammlungen für das Wörterverzeichnis wurden im Jahre 1906 mehrfach durch wertvolle Beiträge aus ungedruckten Quellen gefördert. Solche Beiträge sind insbesondere eingegangen von den HH. Dr. Gümbel in Nürnberg, Oberst a. D. Freiherrn von Guttenberg in Würzburg, Oberst a. D. Freiherrn von Handel-Mazzetti in Linz, Dr. Heerwagen in Nürnberg, Dr. Königer in München, Dr. Vogt und Dr. Vigener in Gießen und Archivdirektor Hofrat G. Winter in Wien. Die Benutzung für die Zwecke des Wörterbuches wurde gestattet von dem steiermärkischen Landesarchiv, das sein reichhaltiges UrkundenSachregister zur Verfügung stellte, von dem oberösterreichischen Landesarchiv, das ebenfalls die Durchsicht seiner Register erlaubte, und von der Hofbibliothek zu Wien (ungedrucktes Vokabular aus dem 18. Jahrhundert). Durch die Vermittlung des Hrn. Hofrats Prof. Dr. Luschin von Ebengreuth wurde eine größere Anzahl von Exzerpten aus dem steiermärkischen Landesarchiv durch Hrn. Dr. Hradil aus den Originalurkunden ergänzt. Von den althochdeutschen Glossen (Steinmeyer und Sievers) wurden die beiden ersten Bände in Angriff genommen. Besonders wertvoll ist, daß nunmehr auch die von Hrn. Dr. Krammer hergestellten Exzerpte aus der Lex Salica und die von Hrn. Willy Ernst übernommenen Auszüge aus dem Schwabenspiegel (nach dem von Hrn. Geh. Hofrat von Rockinger freundlichst zur Beputzung äberlassenen Grundtext seiner in Arbeit befindlichen Ausgabe) vorliegen. Die noch ausstehenden Exzerpte aus dem schwäbischen Lehnrecht werden in kurzem ebenfalls eingeliefert werden. Von der Lex Salica sind die Wörter der malbergischen Glosse nur da aufgenommen, wo sie nach Form und Bedeutung erkennbar erscheinen; dagegen wurden die nur durch Konjektur zu erschließenden Wörter (Kern, van Helten, J. Grimm) unberücksichtigt gelassen. Unter den unten verzeichneten Quellen befindet sich wieder eine große Zahl, deren Exzerpierung der österreichischen Kommission zu verdauken ist. Die Beiträge der schweizerischen Kommission haben eine dankenswerte Bereicherung durch die Exzerpierung des schweizerischen Idiotikons erfahren. 
Verzeichnis der im Jahre 1906 ausgezogenen Quellen.

(Die Beiträge der schweizerischen Kommission sind mit *, die der österreichischen mit * bezeichnet.)

Althochdeutsche Glossen, gesammelt und bearbeitet von E. Steinmeyer und E. Sievers (begonnen): Dr. v. Künßberg.

Analectes pour servir à l'histoire ecclésiastique de la Belgique, IXXXIX: Prof. des Marez, Brüssel.

Baden-Baden, Erbrecht, 15. Jh., ZGOR. 42, $138 \mathrm{ff}$ : Dr. Wahl und Referendär R. Kohler, Heidelberg.

Bad en, Erklärung der Badener zur Vereinbarung der Städte Pforzheim, Ettlingen, Durlach v. J. 1482, ZGOR. 42, 144: Dr. Wahl und Referendär R. Kohler.

Baden, Markgrafschaft, Erbrechtsreform 1464-82, ZGOR. 42, $140 \mathrm{ff}$ : Wahl und R. Kohler.

**Beiträge zur Kunde steirischer Geschichtsquellen, hrsg. v. hist. Ver. f. Steierm., 12. und 13. Jahrgg.: stud. jur. Bayer (Seminar von Schwind).

Beschreibung des Amts Gotha, 17. Jh.: Prof. His, Königsberg.

Böhmer, Acta Imperii (SchluB): Rechtskandidat Joseph, Darmstadt.

Breda, Oude rechtsbronnen der stad, uitg. door Bezemer, 's Gravenhage 1892 (Oude vaderlandsche Rechtsbronnen, I. Reeks, Nr. 14): Prof, van Vleuten, Lausanne.

Bruchsal, Rechtsquellen von (Oberrhein. Stadtrechte I, 843 ff.): Schröder, Dr. Becker, Mannbeim.

Burg, Landrecht von (um 1300), Neue Mitteil. a. d. Geb. hist. antiqu. Forschungen 11, $159 \mathrm{ff}$ : : Assessor Dr. E. Behre, Berlin.

Burghausen, Geschichte d. Stadt B., von J. G. B. Huber 1862: Rechtskandidat Joseph.

Cartula ire d'Afflighem, publié par E. de Marneffe (fasc. 1-5 der Analectes pour servir à l'histoire ecclésiastique, II e section), 18941901: Prof. des Marez, Brüssel.

Cartulaxium der abdy van St. Michiels te Antwerpen (Bijdragen tot de geschiedenis van het hertochdom Brabant 1906): Prof. des Marez, Brüssel.

Cartulaire de l'abbaye de Saint-Trond, publ. par Ch. Piot, 2 vols. Bruxelles 1870-74: Prof. des Marez, Brüssel.

Chroniken der deatschen Städte I-III: Dr. Schmeidler, Berlin.

${ }^{* *}$ Codex Austriacus (Fortsetzung) I-V: Dr. Franz Leifer und stud. jur. Rudolf Zankll (Seminar von Schwind).

Codex diplomaticus Saxoniae regiae II. 1. 2. (Meißen). 14. (Freiberg): Dr. G. Lehnert, Gießen.

Codex Dunensis sive diplomatum et chartarum medii aevi amplissima collectio, ed. baro Kervijn de Lettenhove. Bruxelles 1875: Prof. des Marez, Brïssel.

Coutumes des pays et comté de Flandre. Quartier de Gand. X. Cout. de la seigneurie de Saint-Pierre-les-Gand par D. Kerken. Bruxelles 1905: Prof. des Marez, Brüssel.

Co utume de Scheldewindeke (Bulletin de la comm. roy. des anc. lois de Belgique, VII, 1906): Prof. des Marez, Brüssel.

Danziger Willkür I $(1385-1455)$. O. Günther, Zwei unbek. altpreuß. Willküren. Zeitschr. d. westpreuß. Gesch. Ver. 48: Privatdozent Dr. Koehne, Berlin.

Danziger Willk ür II (Mitte 15. Jhs.) P. Simson, Gesch. der Danziger Willkür, Quellen und Darstellungen zur Gesch. Westpreußens, hrsg. v. Westpreuß. Ver. III. Danzig 1904: Privatdozent Dr. Koehne, Berlin.

Durlach, Schreiben des Schultheißen v. 1482, ZGOR. 42, 142 : Dr. Wahl und Referendär R. Kohler. 
Durlacher Erbrecht, 15. Jh., ZGOR. 42, 133: Dr. Wahl und Referendär R. Kohler.

Erbbuch des Amts Tenneberg (1505): Prof. His, Königsberg.

Ettlinger Erbrecht, 15. Jh., ZGOR. 42, $135 \mathrm{ff}$.: Schröder und Kohler.

Ey b, Des Ritters Ludwig v. Eyb des Älteren Aufzeichnungen über das kaiserl. Landgericht des Burggraftums Nürnberg. Von Wilh. Vogel, Erlangen 1867: Referendar Willy Ernst, Berlin.

Frauenstadt, Drei Malefizbücher. Z. f. d. ges. Strafrechtswissenschaft 23, $269 \mathrm{ff}$; Rechtskandidat Joseph.

Freising, Die Traditionen des Hochstifts Freising I (744-926), hrsg. v. Th. Bitterauf, München 1905: Schröder.

Fruin, De oudste rechten der stad Dordrecht en van het baljuwschap van Znidholland, uitg. door J. A. Fruin. 's Gravenhage 1882: Amtsrichter Dr. Boden, Hamburg, und Prof. des Marez, Brüssel.

Goor, Stadtregt (Ende 14. Jhs.), Overijsselsche Stad-, Dijk- en Markeregten I, 3: Prof. des Marez, Brüssel.

Hannoversche Stadtkundigung 1544. Pufendorf, Observationes IV. app. $220 \mathrm{ff}$ : Dr. Becker, Mannheim.

HasseIt (Utrecht), Stadregt. Overijsselsche Stad-, Dijk- en Markeregten I, 4: Prof. des Marez, Brüssel.

Heilbronn, Urkundenbuch der Stadt, I, bearb. v. Knupfer. Württembergische Geschichtsquellen V. Stuttgart 1904: Privatdozent Dr. Leop. Pereis und stud. theol. Fackler.

** Meier Helmbrecht ron Wernher dem Gartenaere. Panzer, Altdeutsche Textbibliothek Nr. 11. Halle a. S. 1902: Hr. Anton Kraus, Prag.

Hennebergisches Urkundenbuch. Meiningen 1842-77. IV und V: Prof. His, Königsberg.

**Herrgott, M., Monumenta augustae domus Austriacae. Wien 1750-73: stud. Viktor v. Renner (Seminar von Schwind).

**Hohenberg, Der Habsburgische Ottobert. Erfurt 1664: stud. phil. Hornung, Wien.

**H o rma y r, J. v., Wien, seine Geschichte und seine Denkwürdigkeiten. Wien 1823 ff.: stud. Ettel (Seminar von Schwind).

Jena, Urkundenbuch der Stadt. II. Bd. (Thür. Gesch. Qu. VI, 2): stud. jur. Straub, Heidelberg.

Ingolstäd ter Refetbuch (17. Jh.): Dr. Hradil, Graz.

Jordan, Rich., Eigentümlichkeiten des anglischen Wortschatzes. 1905: Dr. Wahl.

**Kaltenbaek, J. P., Die Pan- und Bergteidingbücher in Österr. unter d. Enns. Wien 1846 f.: Dr. Franz Leifer, Wion.

Kampen, Boek van Rechten der Stad K. (Overijsselsche Stad-, Dijken Markeregten I, 1. 2): Prof. des Marez, Brüssel.

**Kärnter Landhand feste 1610: Prof. P. Puntschart, Graz.

Pfaffe Konrad, Rolandslied, hrsg. v. Bartsch. Leipzig 1874: Dr. Kotzenberg, Berlin.

**Ku z z, Franz, Österreich unter Friedr. d. Sch., Albr. IIr., Albr. d. Lahm., Rudolf IV., Ottokar u. Albr.: stud. jur. A. Kohut, Wien.

Pfaffe Lamprecht, Alexanderlied, hrsg. v. Kinzel. Halle 1884: Dr. Kotzenberg, Berlin.

H. v. Lan genstein, Martina (1293), hrsg. v. Keller (Liter. Ver. Stuttg. Nr. 38, 1), 1856: Dr. Arthur Müller, Berlin.

Lehnbuch Friedrichs des Strengen 1349/50, hrsg. v. Lippert und Beschorner. Leipzig 1903: Prof. His, Königsberg.

Lex Salica, ed. Hessels und Kern. London 1888: Dr. Mario Krammer, Berlin.

Limburger Chronik, hrsg. v. Wyss, MG. Script. qui vern. lingua usi sunt IV 1, 25 ff.: Privatdozent Dr. Leop. Pereis. 
Lübeck, Urkundenbuch der Stadt, IV. VII : Referendar Ruben, Berlin.

Matthijssen, Rechtsboek v. d. Briel (Oude vaderlandsche rechtsbronnen, I. Reeks Nr. 1). s'Gravenhage 1880: Prof. van Vleuten, Lausanne.

Michelsen, Rechtsdenkmale aus Thüringen. Jena 1863 (Schluß): Reichsarchivsekretär Oberseider, München.

Mitteilungen der grh. badischen historischen Kommission Nr. 7-9, 24-26: Dr. Hopf, Freiburg i. B.

Monumenta Boica, Bd. 45 (episcop. Wirziburg.). Reichsarchivsekretär Oberseider, München.

Monumenta Germaniae historica, Constitutiones II: Dr. Ernst Perels, Berlin. MG. Constitutiones III : Dr. Stengel, Berlin, und Prof. His, Königsberg.

**N ekrologium des ebemaligen Augustiner-Chorherrenstifts St. Pölten (Fontes rer. Austr. Diplom. et acta 21, 2) : stud. jur. Kerschbaumer, Wien.

Nikolaus v. Jeroschin, Deutschordenschronik, hrsg. Strehlke 1861 (Script. rer. Prussic. 1): Dr. W. Ziesemer, Berlin.

Obergrombach, Rechtsquellen von. Oberrhein. Stadtrechte I, $983 \mathrm{ff}$ : Schröder.

** Ofner Stadtrecht, hrsg. v. Michnay u. Lichner. Preßburg 1845: Dr. v. Künßberg.

Ordonnances des Pays-Bas (Recueil des ord.), II e série, 1. u. 2.: Prof. des Marez, Brüssel.

**Ö sterreichische Urbare I, 1: Die landesfürstl. Urbare Nieder- und Oberösterreichs (13. und 14. Jh.), hrsg. W. Levec u. Dopsch. Wien 1904: Dr. F. Leifer (Seminar von Schwind).

Pardessus, J. M., Diplomata, chartae, epistolae ..... ad res GalloFrancicas spectantia ..... I. II. Paris 1843: Dr. v. Künßberg.

Philippsburg (Udenheim); Rechtsquellen von (Oberrhein. Stadtrechte I, $951 \mathrm{ff}$.): Schröder.

Pf'orzheimer Erbrecht, 15. Jh., ZGOR. 42, 134 : Schröder und Referendär R. Kohler.

Pforzheim, Ettlingen, Durlach, Vereinbarung v. 1482, ZGOR. 42, 143: Dr. Wahl und Referendär R. Kohler.

**Pleier, Tandareis n. Flordibel, hrsg. Khull. Graz 1885: stud. phil. Freud, Wien.

Popovich, Vocabula Austriaca et Styriaca. Handschr. d. Wiener Hofbibl.: Dr. v. Künßberg.

Popovich, Variae. Handschr. d. Wiener Hofbibl.: Dr. v. Künßberg.

Ratzeburg, Reformation 1582 (Pfufendorf Obs. IV. app. $232 \mathrm{ff}$.): Dr. Becker.

** R a u ch, Rerum Austriac. Scriptores. Wien 1793 f.: W. Hanausek, Wien.

Rothenberg, Rechtsquellen von (Oberrhein. Stadtrechte I, 945ff.): Schröder.

Reichstagsakten, Ältere Reihe. 6. und 9. Bd.: Privatdozent Dr. E. Vogt und Dr. Vigener, Gießen.

Salb ücher (ungedruckte) der Staatsarchive von Weimar u. Marburg: Prof. His, Königsberg.

** Scheyb, Theresiade. Wien 1746: stud. phil. Hornung, Wien.

**S chrötter, Abhandl. z. österr. Staatsrecbte. 3 Bde.: stud. jur. Kohut (Seminar von Schwind).

**v. Schwind u. Dopsch, Ausgewählte Urkunden zur Verfassungsgeschichte der deutsch-österr. Erblande im Mittelalter. Innsbruck 1895: stud. Victor v. Renner (Seminar von Schwind).

Schwabenspiegel nach dem für Rockinger gedruckten Handexemplar; Artikelfolge und Vergleichung mit Lassberg nach L. v. Rockinger, Zu HSS. der jüngeren Gestalt des kaiserlichen Land- u. Lehenrechts, 
Abh. d. Münchener Akademie der Wissensch. III. Kl, 22. Bd. 3. Abt. München 1902: Referendar Willy Ernst, Berlin.

Siegener Erbrecht u. Schöffenurteile: Dr. E. Behre, Berlin.

Sint-Truyen, Gewoonten, vryheden en privilegien der stad (14. eeuw.). Gent o. J. Maatschappy der Vlaamsche Bibliophilen. 2. Serie, Nr. 3: Prof. des Marez, Brüssel.

* Sta ub-Tobler, Schweizerisches Idiotikon. I. II.: Dr. F. Balsiger.

Stoinbach, Rechtsquellen von (Oberrhein. Stadtrechte 1, 988ff.): Schröder.

Steirisches Landesarchiv. Urkunden-Sachregister (13. und 14. Jh.): Dr. v. Künßberg und Dr. Hradil, Graz.

** Stricker, Daniel von dem blühenden 'Tal (German. Abhandl. v. K. Weinhold, lirsg. v. F. Vogt, Heft 9): stud. phil. Meznik, Wien.

Sumerlaten. Mittelhochdeutsche Glossen aus den Hss. der k. k. Hofbibliothek zu Wien, hrsg. von Hoffmann v. Fallersleben, 1834: Dr. Wahl.

**'Teuerdank, ed. Goedeke (Deutsche Dichter des 16. Jbs. Bd. 10): stud. phil. Meznik, Wien.

** Urkundenbuch des Landes ob der Enns, hrsg. v. Museum FranciscoCarolinum in Linz. Bd. I-III: Dr. Bilger, Wien.

Utrecht, De middeleeuwschen rechtsbronnen der stad, uitg. door S. Muller. I. II. 's Gravenhage 1883 (Oude vaderlandsche rechtsbrounen 1. Reeks Nr. 3): Prof. des Marez, Brüssel.

Waldkirch, Stadtrecht v. J. 1587, hrsg. v. H. Maurer: stud. jur Straub.

Buch Weinsberg, Kölner Denkwürdigkeiten, bearb. v. Höhlbaum. II. (vollendet): Dr. Wahl.

**Wiener Haupt- und Staatsaktionen, von Karl Weiß. Wien 1854: stud. phil. Zellweker, Wien.

** Worms, Schwazer Bergbau im 15. Jh. Wien 1904: Dr. F. Leifer, Wien (Seminar von Schwind).

Zeitschrift f. Geschichte des Oberrheins. N. F. 19: Dr. Hopf, Freiburg i. B.

\section{Preisaufgaben der Rubenow-Stiftung.}

I. Die Stellung des deutschen Richters zu dem Gesetz seit dem Ausgang des 18. Jahrhunderts.

Es ist zu erforschen, wie sich seit dem Einsetzen der Kodifikationen bis auf die Jetztzeit die Wissenschaft, die Gesetzgebung und die Gerichtspraxis zu dem Problem gestellt haben, ob der Richter nur zur Anwendung der Gesetze oder auch zur Ergänzung von Gesetzeslücken resp. sogar zur Abänderung von Gesetzesbestimmungen berufen sei. Für die Gerichtspraxis ist zunächst festzustellen, inwieweit sie im tatsächlichen Erfolge zu Ergänzungen und Änderungen der Gesetze gelangt ist: des weiteren aber auch, ob sie solche rechtschöpferische Tätigkeit nur unbewußt (im Glauben, das Gesetz lediglich auszulegen) oder auch bewußt geübt, und welche Methoden sie dabei befolgt hat.

Als Forschungsgebiet kommen die Verhältnisse in Deutschland (und speziell in Preußen) in Frage. Aber Ausblicke auf die franzö- 